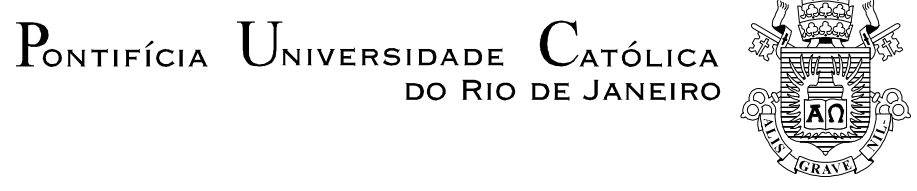

Breno de Castro Vieira

Favoritism and Informational Effects in Public Procurement

Auctions

Dissertação de Mestrado

Thesis presented to the Postgraduate Program in Economics of the Departamento de Economia,PUC-Rio as partial fulfillment for the degree of Mestre em Economia

Adviser: Leonardo Rezende 


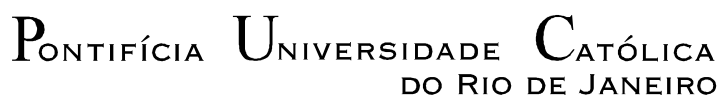

\title{
Favoritism and Informational Effects in Public Procurement
} Auctions

Thesis presented to the Postgraduate Program in Economics of the Departamento de Economia,PUC-Rio as partial fulfillment for the degree of Mestre em Economia

\author{
Leonardo Rezende \\ Adviser \\ Puc-Rio \\ Vinicius do Nascimento Carrasco \\ Puc-Rio
}

Humberto Moreira EPGE/FGV

Prof. Mônica Herz

Coordinator of the Centro de Ciências Sociais - PUC-Rio

Rio de Janeiro,March 30 th 2011 
All rights reserved

\section{Breno de Castro Vieira}

Undergraduate degree in Economics from PUC-Rio in 2008, and Master's degree in Economics from PUC-Rio in 2011. Main areas of interest include Microeconomic Theory, Industrial Organization, and Game Theory. Currently a PhD student at the Stanford University Graduate School of Business.

Bibliographic data

Vieira,Breno de Castro

Favoritism and Informational Effects in Public Procurement Auctions/ Breno de Castro Vieira; adviser: Leonardo Rezende 2011.

40 f. : il. ; $30 \mathrm{~cm}$

Dissertação (Mestrado em Economia)-Pontifícia Universidade Católica do Rio de Janeiro, Rio de Janeiro, 2011.

Inclui bibliografia

1. Economia - Teses. 2. Leilões 3. Compras públicas 4. , Licitações 5. Teoria dos Jogos 6 . Favoritismo I. Rezende, Leonardo. II. Pontifícia Universidade Católica do Rio de Janeiro. Departamento de Economia. III. Título. 


\section{Agradecimentos}

Em primeiro lugar, agradeço aos meus pais Geraldo Vieira Filho e Maria de Fátima de Castro Vieira por todas as oportunidades e a formação, bem como os meus irmãos Guilherme e Fernando Vieira.

Agradeço também a Leonardo Rezende, pelo privilégio de tê-lo como orientador. Suas contribuições como professor e orientador foram fundamentais para minha formação e para a elaboração da dissertação. Agradeço também os comentários de extrema importância de Vinícius Carrasco durante todo o processo de pesquisa.

Agradeço aos amigos e colegas de mestrado, particularmente Rodrigo Adão, Pablo Salgado, e Michel Azulai pelas extensas conversas sobre o tema, bem como Fernando Roriz, Arthur Bragança, Artur Manoel Passos e Clarissa Gandour.

Por fim, agradeço à CAPES pelo apoio financeiro e ao Departamento de Economia pela formação durante os últimos oito anos. 


\section{Resumo}

Vieira, Breno de Castro. Favoritism and Informational Effects in Public Procurement Auctions. Rio de Janeiro, 2011. 40p. Dissertação de Mestrado - Departamento de Economia, Pontifícia Universidade Católica do Rio de Janeiro.

O trabalho desenvolve um modelo de licitações públicas com favoritismo e uma regra de decisão em dois estágios. Devido à existência de favoritismo, a qualidade mínima exigida pela agência para aprovar um projeto é determinada de forma a beneficiar uma das firmas. Mostramos que, mesmo supondo simetria ex-ante entre as firmas participantes e independência entre as distribuições de custo e qualidade, as ofertas de preço serão assimétricas em equilíbrio. Em particular, a firma beneficiada escolhe preços maiores do que as demais para um dado nível de custo. Além disso, simulações do modelo indicam que revelar o número de participantes qualificados diminui o preço que o governo espera pagar pelo bem.

\section{Palavras-chave}

Leilões, Compras públicas, Licitações, Teoria dos Jogos, Favoritismo 


\begin{abstract}
Vieira, Breno de Castro. Favoritism and Informational Effects in Public Procurement Auctions. Rio de Janeiro, 2011. 40p. M.A. Dissertation - Departamento de Economia, Pontifícia Universidade Católica do Rio de Janeiro.
\end{abstract}

We study procurement auctions under favoritism and a two-stage decision rule. Because of favoritism, the minimum quality required in order to approve a project is endogenously determined to benefit the recipient of the agency's bias. We show that, even with ex-ante symmetry and independence between the distributions of costs and quality, equilibrium bidding behavior will be asymmetric and the favorite firm will bid less aggressively than the others. A mechanism design approach indicates that revealing the number of qualified participants or adopting a second-price rule would decrease expected prices and costs of realizing the project, due to the elimination of the asymmetric bidding behavior. Finally, simulations for the uniformly distributed case show that while adopting one of the alternative rules increases efficiency, it also increases the favorite firm's ex-ante profits and therefore may facilitate collusion between the agency and a firm.

\title{
Keywords
}

Auctions, Public Purchases, Procurement, Game Theory, Favoritism 


\section{Sumário}

1 Introduction 9

2 The Model 11

2.1. Basic Setup and Equilibrium 11

$\begin{array}{ll}\text { 2.2. Beliefs } & 13\end{array}$

2.3. Existence and Equilibrium Characterization 14

$\begin{array}{lr}\text { 2.4. The Mechanism Design Problem } & 18\end{array}$

$\begin{array}{ll}\text { 2.5. Alternative Mechanisms } & 20\end{array}$

2.6. Extension - Correlated quality and cost 25

$\begin{array}{ll}3 \text { Concluding remarks } & 27\end{array}$

4 Appendix $\quad 28$

5 References $\quad 39$ 


\section{Lista de figuras}

Figure 1 - Equilibrium Inverse Bid Functions ( $N=3$ ) 17

$\begin{array}{ll}\text { Figure 2- Equilibrium Inverse Bid Functions (N=5) } & 17\end{array}$

Figure 3 Expected Prices and Cost $(\mathrm{N}=3) \quad 23$

Figure 4 - Expected Prices and Cost $(\mathrm{N}=5) \quad 23$

Figure 5 - Expected Profits conditional on $\widetilde{\boldsymbol{q}}(\mathrm{N}=3) \quad 24$

Figure 6 Expected Profits conditional on $\widetilde{\boldsymbol{q}}(\mathrm{N}=5) \quad 25$ 